\title{
VERY HIGH RESOLUTION RADIOMETER
}

\section{Joseph R. Schulman}

The VHRR or very high resolution radiometer was specifically designed to work on an operational, meteorological satellite, and as such is flying now, successfully producing high-quality visible and infrared images from ITOS-D.

It is a two-channel instrument. The visible channel operates in the 0.6 to 0.7 micron region, the IR in the 10.5 to 12.5 micron band. The device has a resolution in both spectral bands of 0.9260 kilometers from a 1460-kilometer polar orbit, and scans from horizon to horizon. The visible channel operates with scene brightness up to $342,626 \mathrm{~cd} / \mathrm{m}^{2}(10,000$ fL) and uses a silicon photodiode as the detector.

The infrared channel operates from a scene radiance equivalent to temperatures in the range of about 185 to about 315 degrees kelvin, and has a temperature discrimination $(\mathrm{NE} \Delta \mathrm{T})$ of approximately three degrees at 185 kelvin and one degree at 315 kelvin.

These numbers have been demonstrated in tests. We have not yet made measurements from orbit. The infrared channel uses a mercury-cadmium telluride detector, operating at 107 degrees kelvin.

Figure 1 shows an optical schematic diagram of the device. The scanning mirror is canted at 45 degrees, and rotates at $400 \mathrm{rpm}$. The optics is a Dahl-Kirkham mirror, Cassegrain system. A dichroic beamsplitter directs the visible and IR energy into the respective detectors.

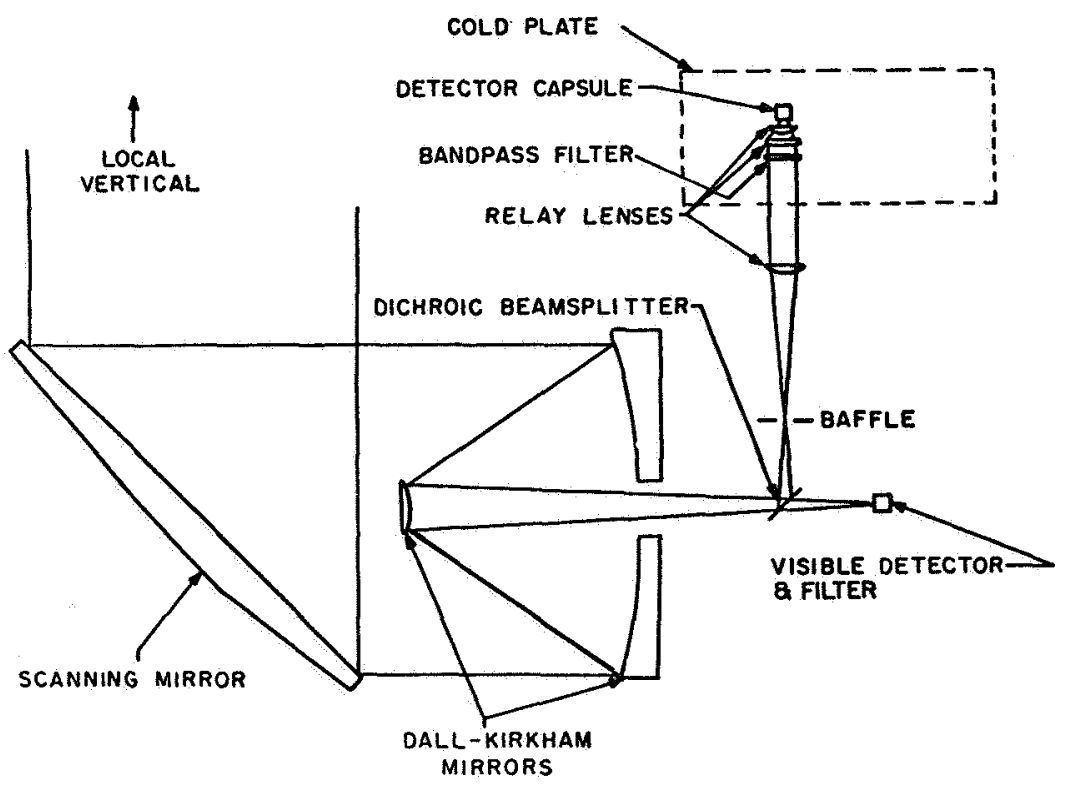

Figure 1. VHRR optical schematic diagram. 
The visible energy goes through the beamsplitter to the visible filter and the silicon photodiode detector. The infrared energy is reflected from the dichroic beamsplitter through a series of relay lenses and a bandpass filter into the IR detector capsule. This is located on the passive radiative cooler, which we will discuss later.

A unique feature of the cooler design is the coupling between the instrument and the cooler; the cooler, represented by the dotted configuration, is through a collimated beam. It gives us leeway in the alignment of the various portions of the instrument.

Figure 2 is a photograph of the instrument. The rotating mirror is here. The optics is hidden. The mirror drive motor is a brushless drive motor servoed to operate at $400 \mathrm{rpm}$. The electronics is distributed, naturally, in the rear of the cooler so you can't see it. However, it is of standard plug-in board construction. The cooler consists of an outer frame, an inner frame, and a cold patch.

The outer frame has second-surface mirrors for cooling. These second-surface mirrors have low absorptivity and high emissivity. The inner frame cooling uses second-surface mirrors; perpendicular to the outer frame is a low emissivity gold.

The white surface is the cold patch, and the string-looking thing is a thermocouple wire. The detector is also shown.

Perhaps the most important technological advance is that the VHRR is the first NASA cooler in orbit operating on an instrument in the 100 degrees kelvin range. It is a two-

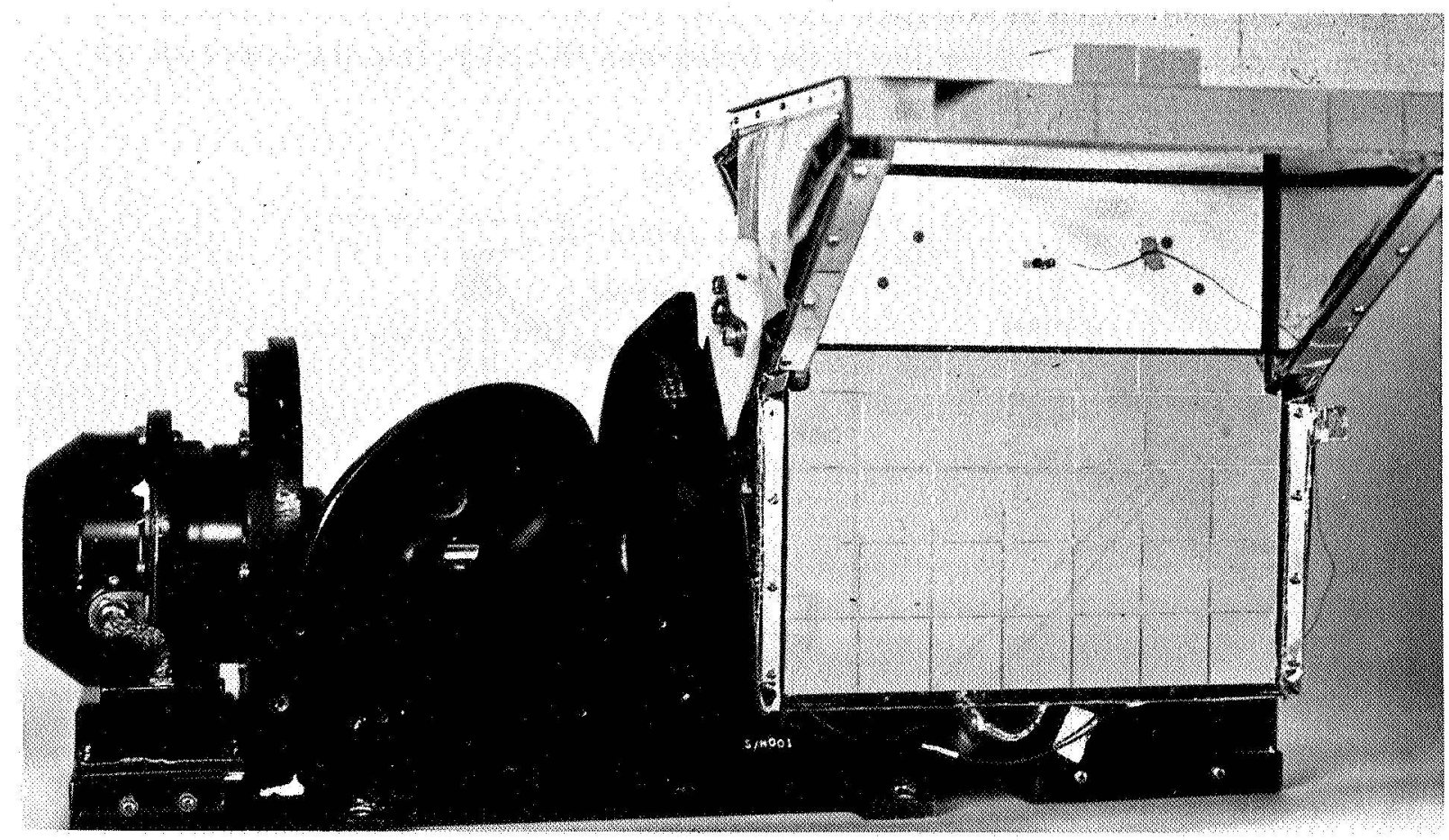

Figure 2. VHRR instrument. 
stage, radiant cooler, with a cooled outer housing, and has a field of view as shown in Figure 3. During the first ten days of operation, we had some anomalous behavior for which we don't yet have the answers.

The design of the cooler is such that the outer frame is supposed to operate in orbit at $214 \mathrm{~K}$, the inner housing at $150 \mathrm{~K}$, and the coldplate, without thermal control, should go down to $95 \mathrm{~K}$.

We use a thermal control servo to obtain the control point of the coldplate to 107 degrees we servo-heat it up, in effect.

In flight, we have obtained the temperatures shown. The main anomaly is the 239 versus 214 degrees difference between the flight and design data. However, the cooler has been designed with sufficient margin to permit within-spec operation of the detector even though we have had higher temperatures on the outer house frame, and slightly higher temperatures on the inner housing.

The coldpatch would operate at 97 degrees kelvin on Serial No. 102 and 101 degrees kelvin on Serial No. 104, if we did not servo the control to 107 kelvin. This gives us an idea of the margin we have; we would like to have more, and we could perhaps obtain an additional few degrees if we could resolve the outer frame anamaly.

Figure 4 would be useful in showing the construction, a cross section of the VHRR cooler.

The inner frame is shown here with a honeycomb panel, second-surface mirrors, the gold that I mentioned, the cold patch, the detector, the filters, and the outer housing. The inner frame and the outer frame are insulated with blankets and with low-conductive standoffs. And, as shown in the far corner, the coldplate is tied to the frame with Kapton bands.

TWO-STAGE RADIANT COOLER WITH COOLED OUTER HOUSING $90^{\circ} \times 188^{\circ}$ FIELD OF VIEW ON ANTI-SUN SIDE OF SPACECRAFT - 1460-km

STAGE

DESIGN

FLIGHT

$\begin{array}{lrrrr} & \text { No. } 102 \text { and } 104 & \text { No. } 102 & \text { No. } 104 \\ \text { OUTER HOUSING } & 214 \mathrm{~K} & 238 \mathrm{~K} & 237 \mathrm{~K} \\ \text { INNER HOUSING } & 150 \mathrm{~K} & 160 \mathrm{~K} & 165 \mathrm{~K} \\ \text { COLD PLATE } & 95 \mathrm{~K} & 97 \mathrm{~K} & 101 \mathrm{~K} \\ & & & \text { [WITH } 107 \mathrm{~K} \text { CONTROL POINTS] }\end{array}$

Figure 3. ITOS-D VHRR radiant cooler, the first NASA $100 \mathrm{~K}$ cooler in orbit. 


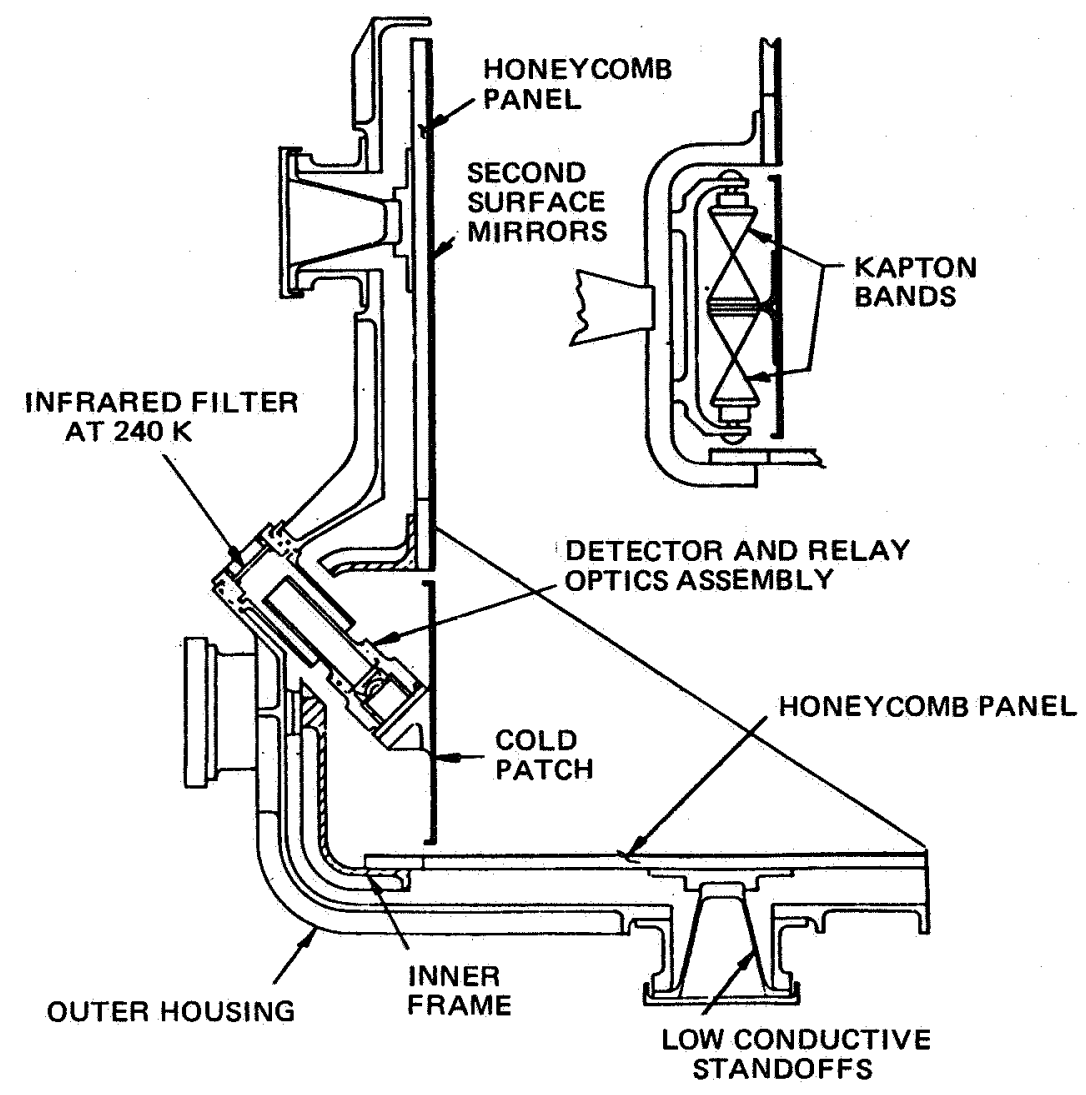

Figure 4. Cross section of VHRR cooler.

I think the visible and IR cloud cover pictures that have been taken, once the ground station was set up properly, were quite startling, really.

\section{MEMBER OF THE AUDIENCE:}

I have seen the picture data, it looks just great. But there was this other question, that I haven't really gotten the answer to. You know there was gain variation, and it wasn't well understood, and you sort of partially explained it, and the instruments we had on board did not exhibit this spacecraft-on, spacecraft-off kind of situation. Have you done enough calibrations in orbit to know that the system is operating stably?

\section{MR. SCHULMAN:}

I really can't answer that right now. Perhaps NOAA might be able to answer it a little better, but they seemed to be quite satisfied with the performance of the instrument. That is all - I can't give you any numbers.

MEMBER OF THE AUDIENCE:

What is the weight of the cooler? And how thick are the walls? 
MR. DONAHOE:

I think it runs about 2 kilograms ( 4 or $5 \mathrm{lb}$ ). The outer housing is a magnesium casting so it is on the order of $1 / 16$ to $1 / 8$. The passive outer housing attaches the cooler to the scanner.

\section{MEMBER OF THE AUDIENCE:}

The overall weight?

MR. SCHULMAN:

It is 10 kilograms $(22 \mathrm{lb})$. 